\title{
Characterization of Interfacial Misfit Array Formation for GaSb Growth on GaAs by Transmission Electron Microscopy
}

\author{
Shenghong Huang*, Ganesh Balakrishnan", and Diana L. Huffaker ${ }^{\&}$ \\ *Department of Earth and Planetary Sciences, University of New Mexico, Albuquerque, NM 87131 \\ ${ }^{\#}$ Center for High Technology Materials, University of New Mexico, Albuquerque, NM 87106 \\ ${ }^{\&}$ California Nano Systems Institute and Department of Electrical Engineering, University of \\ California, Los Angeles, CA 90095
}

Antimonide semiconductors have many potential applications in a wide range of electronic and optoelectronic devices due to unique band-structure alignments, small electron effective mass, and high electron mobility. Recently, a special growth technique involving $90^{\circ}$ interfacial misfit (IMF) dislocations formed using the III-Sb material systems has been demonstrated. [1-2] We show the spontaneous relaxation of the highly mismatched growth of GaSb on GaAs (001) substrate by 2D periodic IMF array, which simultaneously demonstrates very low defect density $\left(\sim 5.4 \times 10^{5}\right.$ defects $/ \mathrm{cm}^{2}$ ). Previously some researchers such as Rocher et. al. have studied the growth of GaSb on GaAs substrate. However, the growth process shown in this letter significantly differs from that of Rocher et. al., where the initial GaSb islands form and subsequent islands coalesce and merge, thus generating many threading dislocations and defects such as $60^{\circ}$ and $90^{\circ}$ dislocations. Because the relaxation in GaSb epilayer via IMF growth mode is instantaneous at $\mathrm{GaSb} / \mathrm{GaAs}$ interface and all of these dislocations are only pure $90^{\circ}$ dislocations, the strain at the interface can be relieved more efficiently compared to the combination of $60^{\circ}$ and $90^{\circ}$ dislocations.

Figure 1 (a) shows a plan-view bright-field TEM image of nominally 3 ML GaSb grown on GaAs substrate. The initial IMF formed along both [110] and [1-10] directions with rather uniform size distribution. The enlarged insert image at the top right corner clearly indicates the perfectly periodic IMF array. A nominally 9 ML GaSb layer deposited on the GaAs substrate is shown in Figure 1(b). The periodicity of the IMF array $(5.6 \mathrm{~nm})$ is confirmed by the high resolution XTEM images in Figure 2, which has agreed with the theoretical values. This periodicity corresponds to exactly one misfit dislocation for every 13 lattice sites of GaSb on 14 lattice sites of GaAs. The Burgers circuit analysis indicates a pure $90^{\circ}$ dislocation with vector $\boldsymbol{a} / 2[110]$.

The bright-field (2-20) two-beam plan-view TEM image of 9 ML GaSb grown on GaAs in Figure 3(a), recorded using $\mathbf{g}=$ condition, reveals the perfect alignment between two neighboring IMF arrays on opposite sides of the small IMF arrays. As these two IMF arrays continue to grow laterally, the distance separating them decreases accordingly, until the two IMF arrays become contiguous. Figure 3(b) shows the bright-field plan-view TEM images of 9 ML GaSb grown on GaAs. The small area $\mathrm{A}$ in Figure 3(b) has no IMF formation but it is enclosed by the periodic IMF array. With continued GaSb deposition, the contiguous IMF array surrounding the coherently strained region A would be expected to envelop region A, thereby replacing its coherent interface with an IMF array contiguous with the surrounding dislocation network.

Using this new growth mode, we have demonstrated a monolithic, electrically-injected, verticallyemitting $\mathrm{GaSb} / \mathrm{AlGaSb}$ light emitting diode emitting at $1.6 \mu \mathrm{m}$ comprised of a hybrid $\mathrm{GaSb} / \mathrm{GaAs}$ 
based structure through the use of this growth mode, the details of which have been discussed elsewhere. [3]

References:

[1] S. H. Huang et al., Appl. Phys. Lett., 88 (2006) 131911.

[2] S. H. Huang et al., Appl. Phys. Lett., 93 (2008) 071102.

[3] M. Mehta et al., Appl. Phys. Lett., 89 (2006) 211110.
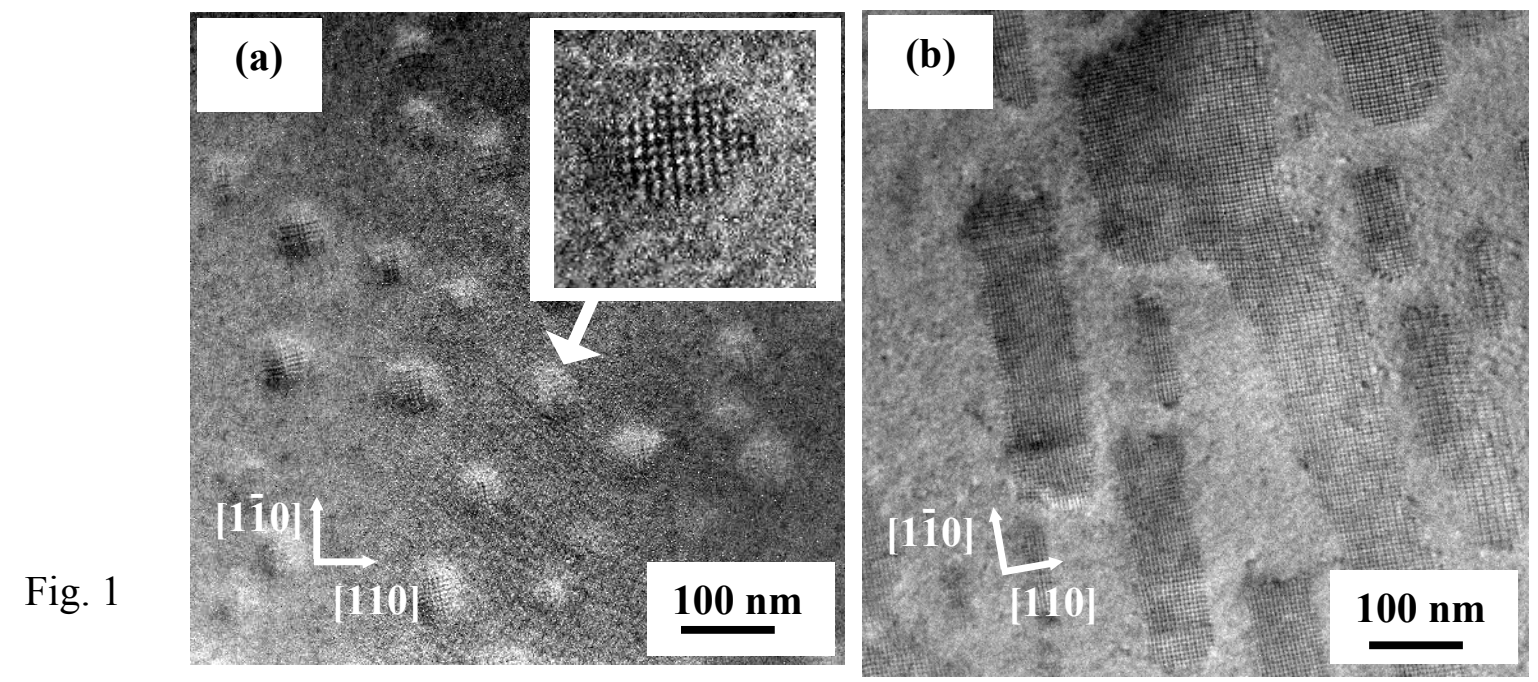

Fig. 2
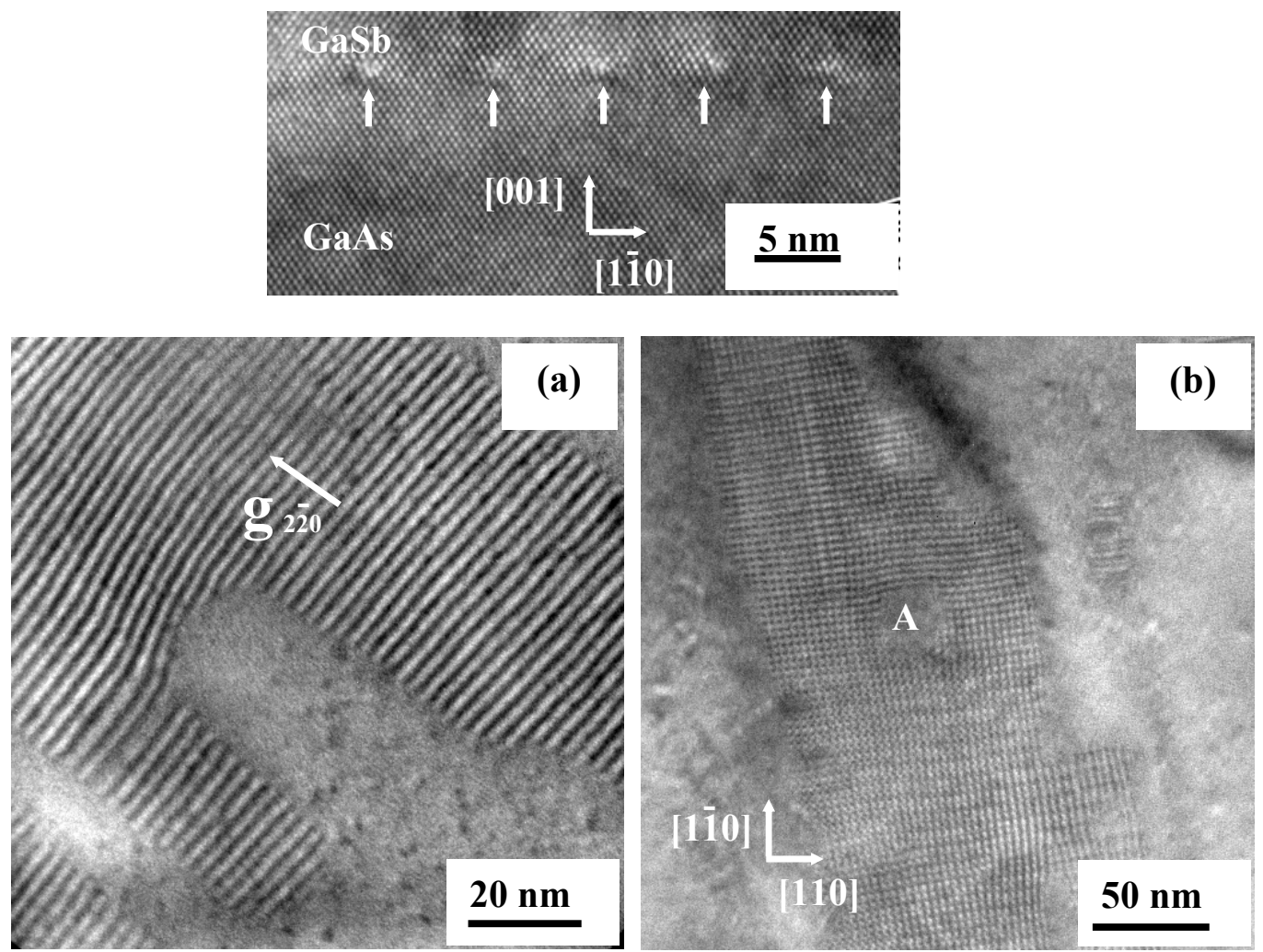